Tomas Ekholm · Rupert L. Frank

\title{
Lieb-Thirring inequalities on the half-line with critical exponent
}

Received December 6, 2006 and in revised form January 22, 2007

Abstract. We consider the operator $-d^{2} / d r^{2}-V$ in $L_{2}\left(\mathbb{R}_{+}\right)$with Dirichlet boundary condition at the origin. For the moments of its negative eigenvalues we prove the bound

$$
\operatorname{tr}\left(-\frac{d^{2}}{d r^{2}}-V\right)_{-}^{\gamma} \leq C_{\gamma, \alpha} \int_{\mathbb{R}_{+}}\left(V(r)-\frac{1}{4 r^{2}}\right)_{+}^{\gamma+(1+\alpha) / 2} r^{\alpha} d r
$$

for any $\alpha \in[0,1)$ and $\gamma \geq(1-\alpha) / 2$. This includes a Lieb-Thirring inequality in the critical endpoint case.

\section{Introduction}

In this paper we consider inequalities for moments of negative eigenvalues of one-dimensional Schrödinger operators. If $V$ is a real-valued function on $\mathbb{R}$ which vanishes at infinity (at least in some averaged sense) then the negative spectrum of $-d^{2} / d x^{2}-V$ consists of discrete eigenvalues of finite multiplicities. The celebrated Lieb-Thirring inequality states that

$$
\operatorname{tr}_{L_{2}(\mathbb{R})}\left(-\frac{d^{2}}{d x^{2}}-V\right)_{-}^{\gamma} \leq L_{\gamma} \int_{\mathbb{R}} V(x)_{+}^{\gamma+1 / 2} d x
$$

holds with a constant $L_{\gamma}$ independent of $V$ if and only if $\gamma \geq 1 / 2$. Here and in what follows, $v_{ \pm}:=\max \{ \pm v, 0\}$ denotes the positive or negative part of $v$. In the non-critical case $\gamma>1 / 2$, bound (1.1) was proved in [11], and the sharp values of the constants $L_{\gamma}$ for $\gamma \geq 3 / 2$ were found in [11] and [3]. The inequality in the endpoint case $\gamma=1 / 2$ was established much later by Weidl [13]. In this case, Hundertmark-Lieb-Thomas [10] determined the sharp value of the constant $L_{1 / 2}$. The sharp constants for $1 / 2<\gamma<3 / 2$ are still unknown.

Egorov-Kondrat'ev [6] studied weighted versions of inequality [1.1. For any $\alpha>0$ they show that

$$
\operatorname{tr}_{L_{2}(\mathbb{R})}\left(-\frac{d^{2}}{d x^{2}}-V\right)_{-}^{\gamma} \leq C_{\gamma, \alpha}^{E K} \int_{\mathbb{R}} V(x)_{+}^{\gamma+(1+\alpha) / 2}|x|^{\alpha} d x
$$

T. Ekholm: Department of Mathematics, Lund University, Box 118, 22100 Lund, Sweden; e-mail: tomase@maths.lth.se

R. L. Frank: Department of Mathematics, Princeton University, Princeton, NJ 08544, USA; e-mail: rlfrank@math.princeton.edu 
holds if and only if $\gamma>(1+\alpha) / 2$. Note that the endpoint case is excluded in contrast to the case $\alpha=0$ and that the potential $V$ can only appear with a power strictly larger than one. Of course, the location of the origin in 1.2 is arbitrary and can be optimized over.

Let us turn to half-line Schrödinger operators $-d^{2} / d r^{2}-V$ in $L_{2}\left(\mathbb{R}_{+}\right)$where $\mathbb{R}_{+}=$ $(0, \infty)$. Throughout we impose a Dirichlet boundary condition at the origin. By the variational principle, inequalities (1.1) and (1.2) remain valid for these operators with the same constants, i.e.,

$$
\operatorname{tr}_{L_{2}\left(\mathbb{R}_{+}\right)}\left(-\frac{d^{2}}{d r^{2}}-V\right)_{-}^{\gamma} \leq C_{\gamma, \alpha}^{E K} \int_{\mathbb{R}_{+}} V(r)_{+}^{\gamma+(1+\alpha) / 2} r^{\alpha} d r .
$$

For $\alpha>0$ it was shown in [6] that $[1.3$ holds even for smaller values of $\gamma$ than (1.2), namely for all $\gamma>\max \{(1-\alpha) / 2,0\}$. However, the validity in the endpoint case $\gamma=$ $(1-\alpha) / 2$ if $0 \leq \alpha<1$ was left open.

In this paper we shall prove a substantially stronger inequality in this endpoint case. Our main result, Theorem 2.1, says that

$$
\operatorname{tr}_{L_{2}\left(\mathbb{R}_{+}\right)}\left(-\frac{d^{2}}{d r^{2}}-\frac{1}{4 r^{2}}-V\right)_{-}^{\gamma} \leq C_{\gamma, \alpha} \int_{\mathbb{R}_{+}} V(r)_{+}^{\gamma+(1+\alpha) / 2} r^{\alpha} d r
$$

for any $0 \leq \alpha<1$ and $\gamma \geq(1-\alpha) / 2$. Since the eigenvalues of $-d^{2} / d r^{2}-V$ decrease if we subtract $1 / 4 r^{2}$, inequality (1.4) extends $(1.3)$ to the endpoint case. Conversely, it is not difficult to see that both (1.3) and 1.4) fail if $\gamma<(1-\alpha) / 2$ and $0 \leq \alpha<1$ (see Remark 2.5.

The main advantage of (1.4) over (1.3) lies, however, in the Hardy term $1 / 4 r^{2}$. First of all, recall the (sharp) Hardy inequality

$$
\frac{1}{4} \int_{\mathbb{R}_{+}} \frac{|u|^{2}}{r^{2}} d r \leq \int_{\mathbb{R}_{+}}\left|u^{\prime}\right|^{2} d r, \quad u \in C_{0}^{\infty}\left(\mathbb{R}_{+}\right) .
$$

This implies that the operator $-d^{2} / d r^{2}-1 / 4 r^{2}$ is non-negative and that the constant $1 / 4$ is maximal with respect to this property. Now it is illuminating to rewrite (1.4) as

$$
\operatorname{tr}\left(-\frac{d^{2}}{d r^{2}}-V\right)_{-}^{\gamma} \leq C_{\gamma, \alpha} \int_{\mathbb{R}_{+}}\left(V(r)-\frac{1}{4 r^{2}}\right)_{+}^{\gamma+(1+\alpha) / 2} r^{\alpha} d r .
$$

This is an inequality of the same form as 1.3 but with a different right hand side. Inequality (1.6) shows that only the part of the potential which is larger than the Hardy weight is necessary to control negative eigenvalues. In particular, it follows from 1.5 that $-d^{2} / d r^{2}-V$ has no negative eigenvalues if $V(r) \leq 1 / 4 r^{2}$ for all $r$. This is reflected in 1.6 but not in 1.3 .

In the case $\alpha=0$ the right hand side of 1.3 coincides, up to a constant, with the semi-classical phase-space integral

$$
\frac{1}{2 \pi} \iint_{\mathbb{R} \times \mathbb{R}_{+}}\left(k^{2}-V(r)\right)_{-}^{\gamma} d k d r .
$$


However, this semi-classical approximation does not take the repulsive Dirichlet condition at the origin into account. This is achieved by (1.6), which decreases the relevant phase-space integral considerably. Inequality 1.6 can indeed be viewed as an infinite phase-space renormalization.

Note also that the operator $-d^{2} / d r^{2}-1 / 4 r^{2}$ appears as the radial part of the twodimensional Laplacian or the critical $d$-dimensional operator $-\Delta-(d-2)^{2} / 4|x|^{2}$ after the natural change of measure. Hence (1.4) estimates moments of eigenvalues of the operator $-\Delta-(d-2)^{2} / 4|x|^{2}-V(|x|)$ in $L_{2}\left(\mathbb{R}^{d}\right)$ corresponding to angular momentum zero.

Our interest in inequality (1.4) originates partially from our previous work [7], where we proved a similar inequality in the case $\alpha \geq 1$ and $\gamma>0$. This was the main tool to extend the multi-dimensional version of (1.1) in the same way as (1.4) extends (1.3). Note, however, that in these considerations the endpoint case $\gamma=0$ is naturally excluded. We also mention the recent alternative proof [9] of the main result of [7].

The proof of $(1.4)$ in the endpoint case $\gamma=(1-\alpha) / 2$ encounters several difficulties. The proof in [10] of [1.1] for $\gamma=1 / 2$ relies heavily on the translation invariance of the whole-line operator. The earlier proof of [13] does so too, but to a lesser extent, and its generalization to our non-translation invariant setting requires additional ideas both on a conceptual and on a technical level. One crucial ingredient in our proof is the combination of Neumann bracketing with the ground-state representation. Despite this (certainly non-optimal) approach we obtain reasonable values for the constant $C_{\gamma, \alpha}$ in (1.4). In the important special case $\alpha=0, \gamma=1 / 2$ we work out upper and lower bounds which differ by less than a factor 2.25 .

In the final section of this paper we show how our main result can be applied to yield a Lieb-Thirring inequality for the operator associated with the generalized Hardy inequality

$$
\frac{(\sigma-1)^{2}}{4} \int_{\mathbb{R}_{+}} \frac{|u|^{2}}{r^{2-\sigma}} d r \leq \int_{\mathbb{R}_{+}} r^{\sigma}\left|u^{\prime}(r)\right|^{2} d r
$$

for suitable $u$. We mention in closing that inequality (1.4) was useful when proving LiebThirring inequalities on regular metric trees [8].

\section{Main result}

Let $V: \mathbb{R}_{+} \rightarrow \mathbb{R}$ with $V_{-} \in L_{1, \text { loc }}\left(\mathbb{R}_{+}\right)$and $V_{+} \in L_{p}\left(\mathbb{R}_{+}, r^{\alpha} d r\right)$ for some $\alpha \geq 0$ and some $1 \leq p<\infty$. The Schrödinger operator $-d^{2} / d r^{2}-1 / 4 r^{2}-V$ in $L_{2}\left(\mathbb{R}_{+}\right)$is defined via the closure of the quadratic form

$$
\int_{\mathbb{R}_{+}}\left(\left|u^{\prime}\right|^{2}-\frac{|u|^{2}}{4 r^{2}}-V|u|^{2}\right) d r
$$

on $C_{0}^{\infty}\left(\mathbb{R}_{+}\right)$. (The fact that this form is bounded from below will follow as soon as we have proved 2.1 for, say, all bounded $V$ with compact support.) Our main result is 
Theorem 2.1. Let $\gamma>0$ and $\alpha \in[0,1)$ such that $\gamma+(1+\alpha) / 2 \geq 1$. Then

$$
\operatorname{tr}\left(-\frac{d^{2}}{d r^{2}}-\frac{1}{4 r^{2}}-V\right)_{-}^{\gamma} \leq C_{\gamma, \alpha} \int_{\mathbb{R}_{+}} V(r)_{+}^{\gamma+(1+\alpha) / 2} r^{\alpha} d r
$$

with a constant $C_{\gamma, \alpha}$ independent of $V$. In the special case $\alpha=0, \gamma=1 / 2$ the sharp constant in this inequality satisfies $0.533 \leq C_{1 / 2,0} \leq 1.185$.

Our result can also be stated in terms of the operator $-d^{2} / d r^{2}-V$, defined similarly with a Dirichlet boundary condition at the origin. Then Theorem 2.1 implies (see [7] for a careful argument)

Corollary 2.2. Let $\gamma>0$ and $\alpha \in[0,1)$ such that $\gamma+(1+\alpha) / 2 \geq 1$. Then

$$
\operatorname{tr}\left(-\frac{d^{2}}{d r^{2}}-V\right)_{-}^{\gamma} \leq C_{\gamma, \alpha} \int_{\mathbb{R}_{+}}\left(V(r)-\frac{1}{4 r^{2}}\right)_{+}^{\gamma+(1+\alpha) / 2} r^{\alpha} d r
$$

with the constant $C_{\gamma, \alpha}$ from 2.1.

Remark 2.3. The most important estimate in Theorem 2.1 is that for the critical case $\gamma=(1-\alpha) / 2$ when $V$ appears with the exponent 1 on the right hand side of (2.1). It shows that eigenvalue moments of any order $0<\gamma \leq 1 / 2$ can be estimated linearly in $V$. (For scaling reasons, however, the integral of $V$ now has to include a weight.) This is in sharp contrast to the whole-line case (1.1) and (1.2), where only moments of order $\gamma=1 / 2$ can be estimated linearly, and where moreover the inclusion of a weight does not allow for smaller values of $\gamma$.

Remark 2.4. The operator $H_{0}:=-d^{2} / d r^{2}-1 / 4 r^{2}$ has a virtual level, in the sense that $H_{0}-V$ has a negative eigenvalue for any non-negative $V \not \equiv 0$. This shows immediately that it is impossible to estimate the number of negative eigenvalues of $H_{0}-V$ in terms of a (weighted) $L_{p}$-norm of $V$. In particular, the critical case $\gamma=0$ is excluded in 2.1) for $\alpha \geq 1$. In order to estimate eigenvalue moments of arbitrarily small order $\gamma>0$ in terms of a (weighted) $L_{p}$-norm of $V$ it is necessary that the lowest eigenvalue $\lambda(\beta)$ of $H_{0}-\beta V$ disappears faster than any polynomial as $\beta \rightarrow 0+$. Indeed, $\lambda(\beta)$ is exponentially small in our case (see [7] for details).

Remark 2.5. The condition $\gamma \geq(1-\alpha) / 2$ in Theorem 2.1 is sharp. Indeed, if $\gamma<$ $(1-\alpha) / 2$ then $V$ appears with a sublinear power on the right hand side of 2.1). Hence if we choose a sequence of potentials $V_{n}:=n \chi_{\left(R, R+n^{-1}\right)}$ with $R>0$ arbitrary, then the right hand side of $(2.1)$ tends to zero as $n \rightarrow \infty$. On the other hand, the sequence $-d^{2} / d r^{2}-1 / 4 r^{2}-V_{n}$ converges in norm resolvent sense to the operator $-d^{2} / d r^{2}-$ $1 / 4 r^{2}-\delta_{R}$, which has a negative eigenvalue. (This can be proved along the lines of [4] Theorem 3.2.3].) Hence the limit of the left hand side of 2.1] is positive.

Remark 2.6. The bounds on $C_{1 / 2,0}$ are based on numerical evaluation of Bessel functions. Note that the upper bound differs from the lower bound by less than a factor 2.25. It is remarkable that $C_{1 / 2,0}$ is strictly larger than $1 / 2$, which is the sharp constant in 1.1 
for $d=1$ and $\gamma=1 / 2$. This means that the (repulsive) Dirichlet boundary condition at the origin cannot completely compensate the (attractive) potential $1 / 4 r^{2}$. In particular, we prove that a potential well $V$ situated near a finite $R$ may have a lower ground-state energy than the same well translated to $R=\infty$.

\section{Proof of the Lieb-Thirring inequality}

This section contains the proof of our main result, Theorem 2.1. It will be given in Subsection 3.2 after stating two basic ingredients in Subsection 3.1 .

\subsection{Operators on a finite interval}

Throughout this section we fix a constant $b>0$. We define the operator $H_{b}$ in $L_{2}(b, b+1)$ via the quadratic form

$$
h_{b}[u]:=\int_{b}^{b+1}\left|\frac{d}{d r}\left(\frac{u(r)}{\sqrt{r}}\right)\right|^{2} r d r, \quad u \in H^{1}(b, b+1) .
$$

Note that this can also be written as

$$
h_{b}[u]=\int_{b}^{b+1}\left(\left|u^{\prime}\right|^{2}-\frac{|u|^{2}}{4 r^{2}}\right) d r-\frac{|u(b+1)|^{2}}{2(b+1)}+\frac{|u(b)|^{2}}{2 b} .
$$

It follows that $H_{b}$ acts as $-d^{2} / d r^{2}-1 / 4 r^{2}$ on functions satisfying the natural boundary conditions

$$
u^{\prime}(b)-\frac{u(b)}{2 b}=u^{\prime}(b+1)-\frac{u(b+1)}{2(b+1)}=0 .
$$

As an aside we remark that $H_{b}$ coincides with the two-dimensional Neumann Laplacian in $\left\{x \in \mathbb{R}^{2}: b<|x|<b+1\right\}$ restricted to radially symmetric functions.

For any $k>0$ the resolvent $\left(H_{b}+k^{2}\right)^{-1}$ exists and is an integral operator with kernel $G_{b}(\cdot, \cdot, k)$, i.e.,

$$
\left(\left(H_{b}+k^{2}\right)^{-1} u\right)(r)=\int_{b}^{b+1} G_{b}(r, s, k) u(s) d s,
$$

for $r \in[b, b+1]$ and $u \in L_{2}(b, b+1)$. We shall need

Lemma 3.1. For any $b \leq r \leq b+1$ the function $G_{b}(r, r, \cdot): \mathbb{R}_{+} \rightarrow \mathbb{R}$ is continuous, non-negative and non-increasing.

Using the explicit expression of $G_{b}$ in terms of Bessel functions we shall establish 
Proposition 3.2. Let $k>0$ and $0 \leq \alpha<1$. Then there is a constant $C_{\alpha}(k)>0$ such that for all $b>0$ and $b \leq r \leq b+1$ one has

$$
G_{b}(r, r, k) \leq C_{\alpha}(k) r^{\alpha} .
$$

For $\alpha=0$ and $k=3.555$ one may choose $C_{0}(3.555)=1 / 3$.

The proof of this proposition will be given in Subsection 4.1 below. Now we use the result to estimate the lowest eigenvalue of the Schrödinger operator $H_{b}-V$ on the interval $(b, b+1)$.

Corollary 3.3. Assume that $V \not \equiv 0$ is a non-negative function on $(b, b+1)$ that satisfies

$$
\int_{b}^{b+1} V(r) r^{\alpha} d r \leq C_{\alpha}(k)^{-1}
$$

for some $k>0$, some $0 \leq \alpha<1$ and $C_{\alpha}(k)$ from (3.4). Then the lowest eigenvalue $\lambda$ of the operator $H_{b}-V$ satisfies

$$
-\lambda \leq k^{2} \text {. }
$$

Proof. By a standard approximation argument we may assume that $V$ is continuous. Taking $u(r)=\sqrt{r}$ as a trial function we see that $\lambda<0$. We denote by $N\left(t^{2}\right)$ the number of eigenvalues of $H_{b}-V$ less than $-t^{2}$. By the Birman-Schwinger principle (see, e.g., [5]) we have

$$
N\left(t^{2}\right) \leq \operatorname{tr} \sqrt{V}\left(H_{b}+t^{2}\right)^{-1} \sqrt{V}=\int_{b}^{b+1} V(r) G_{b}(r, r, t) d r .
$$

(Here we used the continuity of $V$ for the evaluation of the trace.) Now we let $t^{2} \rightarrow-\lambda$ from below, recall that the lowest eigenvalue is simple and use dominated convergence for the right hand side. Denoting

$$
F_{b}(t):=\max _{b \leq r \leq b+1} r^{-\alpha} G_{b}(r, r, t)
$$

we find

$$
1 \leq \int_{b}^{b+1} V(r) G_{b}(r, r, \sqrt{-\lambda}) d r \leq F_{b}(\sqrt{-\lambda}) \int_{b}^{b+1} V(r) r^{\alpha} d r .
$$

Combining this with assumption (3.5) we arrive at

$$
C_{\alpha}(k) \leq F_{b}(\sqrt{-\lambda}) .
$$

On the other hand, Proposition 3.2 is equivalent to $F_{b}(k) \leq C_{\alpha}(k)$. Since $F_{b}$ is a nonincreasing function by Lemma 3.1. we conclude that $\sqrt{-\lambda} \leq k$, as claimed.

The second ingredient in the proof of Theorem 2.1 is the following Poincaré-Sobolev inequality. 
Proposition 3.4. Let $0 \leq \alpha<1$. Then there exists a constant $S_{\alpha}>0$ such that for all $b>0$ and for all $v \in H^{1}(b, b+1)$ with $\int_{b}^{b+1} v(r) r d r=0$ one has

$$
\max _{b \leq r \leq b+1}|v(r)|^{2} r^{1-\alpha} \leq S_{\alpha} \int_{b}^{b+1}\left|v^{\prime}(r)\right|^{2} r d r .
$$

For $\alpha=0$ the sharp constant is $S_{0}=1 / 3$.

The proof of this proposition will be given in Subsection 4.2 We remark that as $b$ grows the function $\sqrt{r}$ on the interval $(b, b+1)$ becomes 'almost constant', so at least intuitively one recovers the inequality

$$
\max _{0 \leq x \leq 1}|v(x)|^{2} \leq \frac{1}{3} \int_{0}^{1}\left|v^{\prime}(x)\right|^{2} d x, \quad \int_{0}^{1} v(x) d x=0,
$$

which played an important role in [13]. Note that allowing for finite values of $b$ does not increase the constant.

Now we deduce from Proposition 3.4 an integral condition on $V$ that guarantees that the operator $H_{b}-V$ has only one negative eigenvalue.

Corollary 3.5. Assume that $V \not \equiv 0$ is a non-negative function on $(b, b+1)$ satisfying

$$
\int_{b}^{b+1} V(r) r^{\alpha} d r \leq S_{\alpha}^{-1}
$$

for some $0 \leq \alpha<1$ and $S_{\alpha}$ from (3.6). Then the operator $H_{b}-V$ has exactly one negative eigenvalue.

Proof. The existence of a negative eigenvalue has already been established in the proof of Corollary 3.3 To prove the uniqueness we note that in view of Proposition 3.4 we have, for all $u \in H^{1}(b, b+1)$ with $\int_{b}^{b+1} u(r) \sqrt{r} d r=0$, the inequality

$$
h_{b}[u]-\int_{b}^{b+1} V(r)|u(r)|^{2} d r \geq\left(1-S_{\alpha} \int_{b}^{b+1} V(r) r^{\alpha} d r\right) h_{b}[u] .
$$

Since this is non-negative by 3.77, we deduce by the variational principle that $H_{b}-V$ has at most one negative eigenvalue.

\subsection{Proof of the main theorem}

Throughout this section we fix $0 \leq \alpha<1$. Our proof follows and extends the ideas of [13]. We divide it into four steps.

Step 1. It suffices to prove Theorem 2.1 in the case $\gamma=\gamma_{c}:=(1-\alpha) / 2$. Indeed, the case $\gamma>\gamma_{c}$ is already contained in [7] or, alternatively, may be deduced from the result for $\gamma=\gamma_{c}$ by the argument of Aizenman-Lieb [3]. The latter is based on the identity

$$
B_{s, t} \lambda_{-}^{s}=\int_{\mathbb{R}_{+}} \mu^{s-t-1}(\lambda+\mu)_{-}^{t} d \mu, \quad s>t,
$$


with some finite constant $B_{s, t}$ (which can be expressed in terms of the beta function). Using it twice and assuming that the result is proven in the critical case one obtains, for any $\gamma>\gamma_{c}$,

$$
\begin{aligned}
\operatorname{tr}\left(-\frac{d^{2}}{d r^{2}}-\frac{1}{4 r^{2}}-V\right)_{-}^{\gamma} & \leq B_{\gamma, \gamma_{c}}^{-1} \int_{\mathbb{R}_{+}} \mu^{\gamma-\gamma_{c}-1} \operatorname{tr}\left(-\frac{d^{2}}{d r^{2}}-\frac{1}{4 r^{2}}-V+\mu\right)_{-}^{\gamma_{c}} d \mu \\
& \leq B_{\gamma, \gamma_{c}}^{-1} C_{\gamma_{c}, \alpha} \int_{\mathbb{R}_{+}} \int_{\mathbb{R}_{+}} \mu^{\gamma-\gamma_{c}-1}(V(r)-\mu)_{+}^{\gamma_{c}+(1+\alpha) / 2} d \mu r^{\alpha} d r \\
& =B_{\gamma, \gamma_{c}}^{-1} B_{\gamma+(1+\alpha) / 2, \gamma_{c}+(1+\alpha) / 2} C_{\gamma_{c}, \alpha} \int_{\mathbb{R}_{+}} V(r)_{+}^{\gamma+(1+\alpha) / 2} r^{\alpha} d r
\end{aligned}
$$

This is inequality [2.1], and so it remains to prove the result for $\gamma=\gamma_{c}$.

Step 2. Now we begin the main argument. The basic strategy is to divide $\mathbb{R}_{+}$into intervals such that the restriction of $-d^{2} / d r^{2}-1 / 4 r^{2}-V$ to these intervals has at most one negative eigenvalue. The choice of boundary conditions for the restricted operators is essential to achieve this. We choose boundary conditions 3.2 which come naturally with the groundstate representation formula (see $(3.9)$ below).

We may assume that $V \not \equiv 0$ is non-negative and, by standard approximation arguments, that it has compact support in $\mathbb{R}_{+}$. Fix $k>0$ arbitrary and let $\Psi_{\alpha}(k):=$ $\max \left\{S_{\alpha}, C_{\alpha}(k)\right\}$ where $S_{\alpha}, C_{\alpha}(k)$ are the constants from Propositions 3.4 and 3.2 We set $a_{1}:=\min \operatorname{supp} V$ and define a sequence $a_{1}<a_{2}<\cdots$ recursively by

$$
\int_{a_{j}}^{a_{j+1}} V(r) r^{\alpha} d r=\frac{1}{\Psi_{\alpha}(k)\left(a_{j+1}-a_{j}\right)^{1-\alpha}} .
$$

This recursion stops when $a_{N} \geq \max \operatorname{supp} V$. The sequence is always finite since $a_{j+1}-$ $a_{j} \geq\left(\Psi_{\alpha}(k)\|V\|_{L_{1}\left(r^{\alpha} d r\right)}\right)^{-1 /(1-\alpha)}>0$, and it clearly covers supp $V$. We set $a_{0}:=0$ and $a_{N+1}:=\infty$.

As in the previous section, we define operators $L_{j}$ in $L_{2}\left(a_{j}, a_{j+1}\right)$ via the quadratic form

$$
\int_{a_{j}}^{a_{j+1}}\left|\frac{d}{d r}\left(\frac{u(r)}{\sqrt{r}}\right)\right|^{2} r d r
$$

with domain $H^{1}\left(a_{j}, a_{j+1}\right)$ if $1 \leq j \leq N$. If $j=0$ we consider the closure of this form defined on $C_{0}^{\infty}\left(0, a_{1}\right]$. Note that for $u \in C_{0}^{\infty}\left(\mathbb{R}_{+}\right)$one has

$$
\int_{\mathbb{R}_{+}}\left(\left|u^{\prime}\right|^{2}-\frac{|u|^{2}}{4 r^{2}}\right) d r=\int_{\mathbb{R}_{+}}\left|\frac{d}{d r}\left(\frac{u(r)}{\sqrt{r}}\right)\right|^{2} r d r .
$$

The variational principle implies that imposing natural boundary conditions does not increase the operator, i.e., in the sense of quadratic forms

$$
-\frac{d^{2}}{d r^{2}}-\frac{1}{4 r^{2}}-V \geq \bigoplus_{j=0}^{N}\left(L_{j}-V\right) .
$$


Since $L_{0}-V=L_{0} \geq 0$ and similarly for $j=N$, we find that

$$
\operatorname{tr}\left(-\frac{d^{2}}{d r^{2}}-\frac{1}{4 r^{2}}-V\right)_{-}^{(1-\alpha) / 2} \leq \sum_{1 \leq j \leq N-1} \operatorname{tr}\left(L_{j}-V\right)_{-}^{(1-\alpha) / 2}
$$

It remains to estimate $\operatorname{tr}\left(L_{j}-V\right)_{-}^{(1-\alpha) / 2}$ for fixed $1 \leq j<N$. We shall implement a unitary change of variables in order to obtain an operator on an interval of unit length and then apply the results from Subsection 3.1 . We put

$$
l_{j}:=a_{j+1}-a_{j}, \quad b_{j}:=a_{j} / l_{j},
$$

and introduce the unitary operator $\mathcal{U}_{j}: L_{2}\left(a_{j}, a_{j+1}\right) \rightarrow L_{2}\left(b_{j}, b_{j}+1\right)$,

$$
\left(\mathcal{U}_{j} u\right)(r):=\sqrt{l_{j}} u\left(l_{j} r\right) .
$$

One obtains the unitary equivalence

$$
\mathcal{U}_{j}^{-1}\left(H_{b_{j}}-V_{j}\right) \mathcal{U}_{j}=l_{j}^{2}\left(L_{j}-V\right)
$$

where $V_{j}(r):=l_{j}^{2} V\left(l_{j} r\right)$. Note that this potential satisfies

$$
\int_{b_{j}}^{b_{j}+1} V_{j}(r) r^{\alpha} d r=\Psi_{\alpha}(k)^{-1}
$$

by 3.8. The definition of $\Psi_{\alpha}(k)$ together with Corollaries 3.5 and 3.3 implies that $H_{b_{j}}-V_{j}$ has exactly one negative eigenvalue, and that its modulus does not exceed $k^{2}$. Combining this with the above unitary equivalence and using (3.8) once more we obtain

$$
\operatorname{tr}\left(L_{j}-V\right)_{-}^{(1-\alpha) / 2} \leq l_{j}^{-1+\alpha} k^{1-\alpha}=k^{1-\alpha} \Psi_{\alpha}(k) \int_{a_{j}}^{a_{j+1}} V(r) r^{\alpha} d r .
$$

In view of 3.10 this concludes the proof of inequality 2.1.

Step 3. We next prove the upper bound $C_{1 / 2,0} \leq 1.185$. For this we note that the above proof yields

$$
C_{(1-\alpha) / 2, \alpha} \leq \inf _{k>0} k^{1-\alpha} \max \left\{S_{\alpha}, C_{\alpha}(k)\right\} .
$$

If $\alpha=0$ we choose $k=3.555$ and use Propositions 3.2 and 3.4 to get the claimed estimate. See Remark 4.1 concerning this choice.

Step 4. Finally, we prove the lower bound $C_{1 / 2,0} \geq 0.533$. We shall first establish

$$
C_{(1-\alpha) / 2, \alpha} \geq \sup _{R>0} R^{1-\alpha} I_{0}(R) K_{0}(R), \quad 0 \leq \alpha<1 .
$$

For $\beta, R>0$ one can define the operator $-d^{2} / d r^{2}-1 / 4 r^{2}-\beta \delta_{R}$ in a standard way via a quadratic form. It follows from general principles that this operator has at most one negative eigenvalue. Moreover, one easily establishes that for any given $R$ there exists a 
unique $\beta=\beta(R)$ such that the operator has -1 as an eigenvalue. Solving the eigenvalue equation explicitly we obtain

$$
u(r)= \begin{cases}\sqrt{r} I_{0}(r) K_{0}(R), & 0<r<R, \\ \sqrt{r} K_{0}(r) I_{0}(R), & R<r,\end{cases}
$$

and simplifying with the help of the Wroński identity

$$
I_{1}(r) K_{0}(r)+I_{0}(r) K_{1}(r)=1 / r
$$

(see [1, 9.6.15]), we find

$$
\beta(R)=\frac{u^{\prime}(R-)-u^{\prime}(R+)}{u(R)}=\frac{1}{R I_{0}(R) K_{0}(R)} .
$$

By an approximation argument as in Remark 2.5 one easily obtains the lower bound (3.11.

Now assume that $\alpha=0$. Using the asymptotic behavior of $I_{0} K_{0}$ [1, 9.7.5] one finds that $\beta(R) \rightarrow 2$ from below as $R \rightarrow \infty$, hence $C_{1 / 2,0}>1 / 2$. To obtain an explicit estimate on the constant we choose $R=1.1$ and use $\beta(1.1)^{-1}>0.533$ (see [1, Table 9.8]). (Indeed, numerically, one finds that $\beta$ has a global minimum close to 1.075.)

This concludes the proof of Theorem 2.1

\section{The operators on a finite interval}

\subsection{Green's function}

By Sturm-Liouville theory (see, e.g., [14]) we find that the resolvent kernel (3.3) is given by

$$
G_{b}(r, s, k)= \begin{cases}\frac{g_{b}(r, k) g_{b+1}(s, k)}{W_{b}(k)}, & b \leq r \leq s \leq b+1, \\ \frac{g_{b+1}(r, k) g_{b}(s, k)}{W_{b}(k)}, & b \leq s \leq r \leq b+1,\end{cases}
$$

where

$$
\begin{aligned}
g_{c}(r, k) & :=\sqrt{r}\left(I_{1}(c k) K_{0}(k r)+K_{1}(c k) I_{0}(k r)\right), \quad c \in\{b, b+1\}, \\
W_{b}(k) & :=I_{1}((b+1) k) K_{1}(b k)-I_{1}(b k) K_{1}((b+1) k) .
\end{aligned}
$$

Here $I_{n}$ and $K_{n}$ denote the modified Bessel functions of the first and second kinds of order $n$ (see [1, Chapter 9]).

Now we give the simple

Proof of Lemma 3.1. The continuity of $G_{b}(r, r, k)$ in $k$ follows from (4.1) by the continuity of the Bessel functions. Moreover, for any $f \in L_{2}(b, b+1)$ and any $k \geq t \geq 0$ one has

$$
0 \leq\left(\left(H_{b}+k^{2}\right)^{-1} f, f\right) \leq\left(\left(H_{b}+t^{2}\right)^{-1} f, f\right)
$$


Choosing $f$ as an approximate delta-function one easily finds that the resolvent kernel is non-negative on the diagonal and non-increasing in the spectral parameter.

Now we turn to the

Proof of Proposition 3.2. For fixed $k>0$ and $0 \leq \alpha<1$ we define

$$
\begin{aligned}
g_{\alpha}(x, b):= & (b+x)^{-\alpha} G_{b}(b+x, b+x, k) \\
= & (b+x)^{1-\alpha}\left(I_{1}(b k) K_{0}((b+x) k)+I_{0}((b+x) k) K_{1}(b k)\right) \\
& \times\left(I_{1}((b+1) k) K_{0}((b+x) k)+I_{0}((b+x) k) K_{1}((b+1) k)\right) \\
& \times\left(I_{1}((b+1) k) K_{1}(b k)-I_{1}(b k) K_{1}((b+1) k)\right)^{-1}
\end{aligned}
$$

for $x \in[0,1], b>0$. We have to prove that there exists a constant $C_{\alpha}(k)>0$ such that for all $x \in[0,1], b>0$ one has

$$
g_{\alpha}(x, b) \leq C_{\alpha}(k)
$$

We begin with the case $\alpha=0$. Using the asymptotic behavior of the Bessel functions [1, 9.6.7, 9.6.8, 9.7.1, 9.7.2] one finds that, uniformly in $x \in[0,1]$,

$$
g_{0}(x, 0):=\lim _{b \rightarrow 0} g_{0}(x, b)=\frac{x I_{0}(k x)\left(I_{1}(k) K_{0}(k x)+K_{1}(k) I_{0}(k x)\right)}{I_{1}(k)}
$$

and

$$
g_{0}(x, \infty):=\lim _{b \rightarrow \infty} g_{0}(x, b)=\frac{\cosh (k x) \cosh (k(1-x))}{k \sinh k}
$$

Both limiting functions $g_{0}(\cdot, 0)$ and $g_{0}(\cdot, \infty)$ are uniformly bounded on $[0,1]$. Since $g_{0}$ is continuous on $[0,1] \times \mathbb{R}_{+}$we obtain the bound $(4.2)$ for $\alpha=0$.

The statement is proved similarly for $0<\alpha<1$. Indeed, for $b \geq 1$ the statement is weaker than for $\alpha=0$. To treat small $b$ one notices that $g_{\alpha}(x, b) \sim-(b+x)^{1-\alpha} \log (b+x)$ as $(x, b) \rightarrow(0,0)$.

Finally, we give a numerical estimate of $C_{0}(k)$ for the special choice $k=3.555$. The function $g_{0}$ can be maximized numerically on $[0,1] \times[0, \infty)$. (Simple estimates show that one can restrict oneself to a compact subset.) Using MATHEMATICA's Nelder-Mead based method one finds that the maximum is attained at $(x, b)=(1,0)$ and one has

$$
\sup g_{0}=g_{0}(1,0)<1 / 3 \text {. }
$$

Hence $C_{0}(3.555)<1 / 3$.

Remark 4.1. Numerical calculations suggest that the Green's function on the diagonal $G_{b}(r, r, k)$ attains its maximum at the right endpoint $r=b+1$ for any value of $b$ and $k$. This would imply that

$$
\max _{x \in[0,1]} g_{0}(x, b)=g_{0}(1, b), \quad b>0 .
$$


Moreover, one can check that the function $g_{0}(1, \cdot)$ attains its supremum in the limit $b \rightarrow 0$. By 4.3 and (3.12) one finds the value $g(1,0)=I_{0}(k) / k I_{1}(k)$. Hence we believe that the sharp constant in (3.4) for $\alpha=0$ is given by

$$
C_{0}(k)=\frac{I_{0}(k)}{k I_{1}(k)}
$$

Note that the RHS is a decreasing function and that $k=3.555$ in the above proof is chosen (almost) maximal with the property that $I_{0}(k) / k I_{1}(k) \leq 1 / 3$.

\subsection{A Poincaré-Sobolev inequality}

We turn now to the proof of Proposition 3.4 The core is contained in

Lemma 4.2. Let $b>0$ and $b \leq c \leq b+1$. Then for all $v \in H^{1}(b, b+1)$ satisfying $\int_{b}^{b+1} v(r) r d r=0$ one has

$$
|v(c)|^{2} \leq \Phi(b, c) \int_{b}^{b+1}\left|v^{\prime}(r)\right|^{2} r d r
$$

with the sharp constant

$$
\begin{aligned}
\Phi(b, c):= & \frac{1}{4(2 b+1)^{2}}\left(4(b+1)^{4} \log (b+1)-4 b^{4} \log b\right. \\
& \left.-(2 b+1)\left(3+6 b+6 b^{2}-4 c^{2}+4\left(2 b^{2}+2 b+1\right) \log c\right)\right) .
\end{aligned}
$$

Proof. We shall assume $b<c<b+1$. The remaining cases $b=c$ and $b=c+1$ are proved similarly. We consider the functional

$$
F[v]:=\frac{\int_{b}^{b+1}\left|v^{\prime}(r)\right|^{2} r d r}{|v(c)|^{2}}
$$

on the domain

$$
\mathcal{D}[F]:=\left\{v \in H^{1}(b, b+1): \int_{b}^{b+1} v(r) r d r=0, v(c) \neq 0\right\} .
$$

From the compactness of the embedding $H^{1}(b, b+1) \subset C([b, b+1])$ (see, e.g., [2]), it follows that $F$ has a minimizer $v$. We may normalize $v$ by

$$
\frac{1}{v(c)} \int_{b}^{b+1} v^{\prime}(r)^{2} r d r=1
$$


In a standard way we derive the Euler-Lagrange equation $\left(v^{\prime}(r) r\right)^{\prime}=2 r /(2 b+1)$ for $r \in(b, c) \cup(c, b+1)$ and the boundary conditions $v^{\prime}(r)=0$ for $r \in\{b, b+1\}$. We conclude that

$$
v(r)= \begin{cases}D-\frac{b^{2}}{2 b+1} \log r+\frac{r^{2}}{2(2 b+1)}, & b \leq r \leq c, \\ D+\log c-\frac{(b+1)^{2}}{2 b+1} \log r+\frac{r^{2}}{2(2 b+1)}, & c \leq r \leq b+1,\end{cases}
$$

where

$$
\begin{aligned}
D:=\frac{1}{4(2 b+1)^{2}} & \left(4(b+1)^{4} \log (b+1)-4 b^{4} \log b\right. \\
& \left.-(2 b+1)\left(3+6 b+6 b^{2}-2 c^{2}+4(b+1)^{2} \log c\right)\right) .
\end{aligned}
$$

For the minimal value of the functional we obtain $F[v]=v(c)^{-1}=\Phi(b, c)^{-1}$, as claimed.

Proof of Proposition 3.4. For $x \in[0,1], b>0,0 \leq \alpha<1$ we put

$$
\begin{aligned}
\phi_{\alpha}(x, b):= & (b+x)^{1-\alpha} \Phi(b, b+x) \\
= & \frac{(b+x)^{1-\alpha}}{4(2 b+1)^{2}}\left(4(b+1)^{4} \log (b+1)-4 b^{4} \log b\right. \\
\left.-(1+2 b)\left(3+6 b+6 b^{2}-4(b+x)^{2}\right)\right) & \\
& -\frac{2 b^{2}+2 b+1}{2 b+1}(b+x)^{1-\alpha} \log (b+x) .
\end{aligned}
$$

We have to prove that there exists a constant $S_{\alpha}>0$ such that for all $x \in[0,1], b>0$ one has

$$
\phi_{\alpha}(x, b) \leq S_{\alpha} .
$$

First we note that $\phi_{\alpha}$ can be extended continuously to the boundary $\{b=0\}$. Indeed, uniformly in $x \in[0,1]$,

$$
\phi_{\alpha}(x, 0):=\lim _{b \rightarrow 0} \phi_{\alpha}(x, b)= \begin{cases}x^{1-\alpha}\left(-\log x+x^{2}-3 / 4\right) & \text { if } x \neq 0, \\ 0 & \text { if } x=0 .\end{cases}
$$

Similarly, one finds that uniformly in $x \in[0,1]$,

$$
\phi_{\alpha}(x, \infty):=\lim _{b \rightarrow \infty} \phi_{\alpha}(x, b)= \begin{cases}1 / 3-x+x^{2} & \text { if } \alpha=0, \\ 0 & \text { if } 0<\alpha<1 .\end{cases}
$$

Hence the continuity of $\phi_{\alpha}$ implies that 4.5) holds with some finite constant $S_{\alpha}$.

Finally, we turn to the issue of the sharp value of the constant for $\alpha=0$. The proof that $S_{0}=1 / 3$ is elementary but tedious. We shall write $\phi$ instead of $\phi_{0}$. Note that the limit of $\phi(\cdot, b)$ as $b \rightarrow \infty$ implies that the sharp constant cannot be less than $1 / 3$. 
To prove the opposite inequality one checks first that $\phi(0, \cdot)$ is an increasing function with $\phi(0,0)=0$ and $\phi(0, \infty)=1 / 3$. Similarly, $\phi(1, \cdot)$ is an increasing function with $\phi(1,0)=1 / 4$ and $\phi(1, \infty)=1 / 3$. Now we distinguish according to whether $\phi(\cdot, b)$ has a local maximum in $(0,1)$ or not. In the latter case we use the facts mentioned above to get

$$
\max _{x \in[0,1]} \phi(x, b)=\max \{\phi(0, b), \phi(1, b)\} \leq 1 / 3 .
$$

Now consider the case where $x_{0} \in(0,1)$ is a local maximum. Again by the facts mentioned above it suffices to prove that

$$
\phi\left(x_{0}, b\right) \leq 1 / 3
$$

We first claim that one necessarily has

$$
0 \leq x_{0} \leq 1 / \sqrt{6} \text { and } 0 \leq b \leq(1+\sqrt{5}) / 4 .
$$

Indeed, note that

$$
\partial_{x}^{2} \phi_{0}=\frac{1}{(2 b+1)(b+x)}\left(6(b+x)^{2}-\left(2 b^{2}+2 b+1\right)\right) .
$$

Since $x_{0}$ is a local maximum, we conclude that

$$
0 \leq x_{0} \leq \sqrt{\left(1+2 b+2 b^{2}\right) / 6}-b \text { and } \sqrt{\left(1+2 b+2 b^{2}\right) / 6} \geq b,
$$

which is easily seen to imply (4.7).

To proceed, we decompose $\phi(x, b)=\phi^{(1)}(x, b)+\phi^{(2)}(x, b)$, where

$$
\begin{aligned}
\phi^{(1)}(x, b):=\frac{2 b^{2}+2 b+1}{6(2 b+1)}( & -6(b+x) \log (b+x)+6(x+b-1) \\
& \left.+3(x+b-1)^{2}-(x+b-1)^{3}\right) .
\end{aligned}
$$

We can estimate $\phi^{(1)}(x, b) \leq 0$ for all $(x, b)$ satisfying (4.7) (with $x_{0}$ replaced by $x$ ). Now we note that $\phi^{(2)}(\cdot, b)$ is a polynomial of degree three. A tedious but elementary calculation shows that it has a local maximum $x_{1}(b)$ and a local minimum $x_{2}(b)$ satisfying $x_{1}(b)<0<1 / \sqrt{6}<1 / 2<x_{2}(b)$ for $0 \leq b \leq(1+\sqrt{5}) / 4$. Hence we conclude that $\phi^{(2)}(x, b) \leq \phi^{(2)}(0, b) \leq 1 / 3$ for all $(x, b)$ satisfying (4.7) (with $x_{0}$ replaced by $x$ ). This proves 4.6.

Remark 4.3. What we actually have shown in the preceding proof is that the minimizer $v_{*}$ of the problem

$$
\max _{b \leq r \leq b+1}|v(r)|^{2} r \leq \Phi(b) \int_{b}^{b+1}\left|v^{\prime}(r)\right|^{2} r d r
$$

satisfies $\max _{b \leq r \leq b+1}\left|v_{*}(r)\right|^{2} r=\left|v_{*}(b+1)\right|^{2}(b+1)$. The proof of Proposition 3.4 would be simplified if we could prove this a priori. 


\section{A class of half-line operators}

Let $\sigma \in \mathbb{R}$. We consider the quadratic form

$$
h_{\sigma}[u]:=\int_{\mathbb{R}_{+}}\left(r^{\sigma}\left|u^{\prime}(r)\right|^{2}-\frac{(\sigma-1)^{2}|u(r)|^{2}}{4 r^{2-\sigma}}\right) d r
$$

defined on $C_{0}^{\infty}\left(\mathbb{R}_{+}\right)$if $\sigma<1$ and on $C_{0}^{\infty}\left(\overline{\mathbb{R}_{+}}\right)$if $\sigma \geq 1$. The generalized Hardy inequality (1.7) implies that the forms $h_{\sigma}$ are non-negative on their respective domains. (We shall essentially reprove this in the proof of Theorem 5.1.) Moreover, they are closable in $L_{2}\left(\mathbb{R}_{+}\right)$and thus generate self-adjoint operators $H_{\sigma}$. Note that the operator $H_{0}$ coincides with the operator $-d^{2} / d r^{2}-1 / 4 r^{2}$ treated in previous sections.

Our main result in this section are Lieb-Thirring inequalities on the moments of negative eigenvalues of the Schrödinger-type operator $H_{\sigma}-V$.

Theorem 5.1. Let $\gamma>0$. Assume that either

$$
\sigma>2, \quad \alpha \leq-\sigma / 2, \quad \gamma-\frac{1+\alpha}{\sigma-2} \geq 1
$$

or

$$
\sigma<2, \quad \alpha \geq-\sigma / 2, \quad \gamma+\frac{1+\alpha}{2-\sigma} \geq 1
$$

Then

$$
\operatorname{tr}\left(H_{\sigma}-V\right)_{-}^{\gamma} \leq\left|\frac{2}{2-\sigma}\right|^{\frac{2 \alpha+\sigma}{2-\sigma}} C_{\gamma, \frac{2 \alpha+\sigma}{2-\sigma}} \int_{\mathbb{R}_{+}} V(r)_{+}^{\gamma+\frac{1+\alpha}{2-\sigma}} r^{\alpha} d r,
$$

where the constant $C_{\gamma, \alpha}$ is given in Theorem 2.1 .

Finally, let $\sigma=2$ and assume that either $\gamma \geq 1 / 2$ and $\alpha=0$, or $\gamma>0$ and $\alpha>0$ with $\gamma+(1+\alpha) / 2>1+\alpha$. Then

$$
\operatorname{tr}\left(H_{2}-V\right)_{-}^{\gamma} \leq C_{\gamma, \alpha}^{E K} \int_{\mathbb{R}_{+}} V(r)_{+}^{\gamma+(1+\alpha) / 2}|\log r|^{\alpha} r^{-1} d r
$$

where the constant $C_{\gamma, 0}^{E K}=L_{\gamma}$ is given in (1.1) and $C_{\gamma, \alpha}^{E K}$ in 1.2.

Proof. We shall first consider the case $\sigma \neq 2$ and introduce the unitary operators $U_{\sigma}$ in $L_{2}\left(\mathbb{R}_{+}\right)$,

$$
\left(U_{\sigma} u\right)(r):=\left|\frac{2-\sigma}{2}\right|^{1 / 2} r^{-\sigma / 4} u\left(r^{(2-\sigma) / 2}\right) .
$$

We note that the adjoint operators $U_{\sigma}^{*}$ map $C_{0}^{\infty}\left(\mathbb{R}_{+}\right)$into itself and, for $\sigma \geq 1$, map $C_{0}^{\infty}\left(\overline{\mathbb{R}_{+}}\right)$into the form domain of $H_{0}$. Moreover, one easily verifies that

$$
h_{\sigma}[u]=\left(\frac{2-\sigma}{2}\right)^{2} h_{0}\left[U_{\sigma}^{*} u\right] .
$$


This relation, proved initially for $u \in C_{0}^{\infty}\left(\mathbb{R}_{+}\right)$if $\sigma<1$-or for $u \in C_{0}^{\infty}\left(\overline{\mathbb{R}_{+}}\right)$if $\sigma \geq 1-$ extends to the closure of $h_{\sigma}$ and implies that

$$
U_{\sigma}^{*} H_{\sigma} U_{\sigma}=\left(\frac{2-\sigma}{2}\right)^{2} H_{0} .
$$

For given $V$ we define

$$
V_{\sigma}(r):=\left(\frac{2}{2-\sigma}\right)^{2} V\left(r^{2} /(2-\sigma)\right)
$$

and find that

$$
\operatorname{tr}\left(H_{\sigma}-V\right)_{-}^{\gamma}=\left|\frac{2-\sigma}{2}\right|^{2 \gamma} \operatorname{tr}\left(H_{0}-V_{\sigma}\right)_{-}^{\gamma} .
$$

Hence (5.2) follows from our main result, Theorem 2.1.

In the case $\sigma=2$ we define the unitary operator $U_{2}: L_{2}(\mathbb{R}) \rightarrow L_{2}\left(\mathbb{R}_{+}\right)$by $\left(U_{2} u\right)(r):=r^{-1 / 2} u(\log r)$. Similarly to the above one checks that

$$
U_{2}^{*} H_{2} U_{2}=-\frac{d^{2}}{d x^{2}}
$$

and hence with $V_{2}(x):=V\left(e^{x}\right)$,

$$
\operatorname{tr}_{L_{2}\left(\mathbb{R}_{+}\right)}\left(H_{2}-V\right)_{-}^{\gamma}=\operatorname{tr}_{L_{2}(\mathbb{R})}\left(-\frac{d^{2}}{d x^{2}}-V_{2}\right)_{-}^{\gamma} .
$$

Inequality (5.3) now follows from (1.1) and (1.2).

Remark 5.2. Note that the sharp constants $C_{\gamma, 0}^{E K}=L_{\gamma}$ in 5.3 with $\alpha=0$ are known if $\gamma=1 / 2$ or $\gamma \geq 3 / 2$.

Remark 5.3. The method of the previous proof allows one to obtain rather complete information on the behavior of weakly coupled eigenvalues. Assume for simplicity that $V$ is bounded with compact support in $\mathbb{R}_{+}{ }^{1}$ Then $H_{\sigma}-\beta V$ has a negative eigenvalue for all $\beta>0$ if and only if $V \not \equiv 0$ and $\int_{\mathbb{R}_{+}} V(r) r^{1-\sigma} d r \geq 0$. In the case $\int_{\mathbb{R}_{+}} V(r) r^{1-\sigma} d r>0$ there is a unique eigenvalue $\lambda(\beta)$ for all sufficiently small $\beta$, and one has

$$
\begin{array}{rlrl}
\left.\lim _{\beta \rightarrow 0} \beta^{-1}|\log | \lambda(\beta)\right|^{-1} & =\frac{1}{2-\sigma} \int_{\mathbb{R}_{+}} V(r) r^{1-\sigma} d r, & \sigma \neq 2, \\
\lim _{\beta \rightarrow 0} \beta^{-2} \lambda(\beta) & =-\frac{1}{4}\left(\int_{\mathbb{R}_{+}} V(r) \frac{d r}{r}\right)^{2}, & & \sigma=2 .
\end{array}
$$

As in the proof of Proposition 5.1, this assertion is reduced to the operators $H_{0}$ and $-d^{2} / d x^{2}$, for which the assertion is known (see [12] and [7]). One can treat the case where $\int_{\mathbb{R}_{+}} V(r) r^{1-\sigma} d r=0$ in a similar manner, but we omit the details.

\footnotetext{
1 It will be sufficient that $\int_{\mathbb{R}_{+}}|V(r)|^{1+\delta} r^{1-\sigma} d r+\int_{\mathbb{R}_{+}}|V(r)|\left(1+r^{(2-\sigma) \delta / 2}\right) r^{1-\sigma} d r<\infty$ for some $\delta>0$ if $\sigma \neq 2$, and that $\int_{\mathbb{R}_{+}}|V(r)|(1+r) \frac{d r}{r}<\infty$ if $\sigma=2$.
} 
Acknowledgments. This work was partially supported by FCT Portugal, post-doc grant SFRH/BPD/23820/2005, (T.E.), by the Swedish Foundation for International Cooperation in Research and Higher Education (STINT) (R.F.), as well as through the ESF Scientific Programme in Spectral Theory and Partial Differential Equations (SPECT). The authors are grateful to the American Institute of Mathematics for the invitation to the workshop Low Eigenvalues of Laplace and Schrödinger Operators where this problem was brought up. R.F. would like to thank E.H. Lieb and R. Seiringer for the hospitality at Princeton University and for helpful discussions. Remarks by A. Hansson and A. Laptev are gratefully acknowledged.

\section{References}

[1] Abramowitz, M., Stegun, I. A.: Handbook of Mathematical Functions with Formulas, Graphs, and Mathematical Tables. Reprint of the 1972 edition. Dover Publ., New York (1992) Zbl 0643.33001 MR 1225604

[2] Adams, R. A.: Sobolev Spaces. Academic Press, New York (1975) Zbl 0314.46030 MR 0450957

[3] Aizenman, M., Lieb, E.: On semiclassical bounds for eigenvalues of Schrödinger operators. Phys. Lett. A 66, 427-429 (1978) MR 0598768

[4] Albeverio, S., Gesztesy, F., Høegh-Krohn, R., Holden, H.: Solvable Models in Quantum Mechanics. 2nd ed., with an appendix by P. Exner, AMS Chelsea Publ., Providence (2005). Zbl 1078.81003 MR 2105735

[5] Birman, M. Sh., Solomyak, M. Z.: Schrödinger operators. Estimates for number of bound states as function-theoretic problem. Amer. Math. Soc. Transl. (2) 150, 1-54 (1992) Zbl 0756.35057 MR 1157648

[6] Egorov, Yu. V., Kondrat'ev, V. A.: On Spectral Theory of Elliptic Operators. Oper. Theory Adv. Appl. 89, Birkhäuser, Basel (1996) Zbl 0855.35001 MR 1409364

[7] Ekholm, T., Frank, R. L.: On Lieb-Thirring inequalities for Schrödinger operators with virtual level. Comm. Math. Phys. 264, 725-740 (2006) Zbl 1106.81039||MR 2217288

[8] Ekholm, T., Frank, R. L., Kovařík, H.: Eigenvalue estimates for Schrödinger operators on regular metric trees. Preprint, arXiv:0710.5500

[9] Frank, R. L., Lieb, E. H., Seiringer, R.: Hardy-Lieb-Thirring inequalities for fractional Schrödinger operators. J. Amer. Math. Soc., to appear

[10] Hundertmark, D., Lieb, E. H., Thomas, L. E.: A sharp bound for an eigenvalue moment of the one-dimensional Schrödinger operator. Adv. Theor. Math. Phys. 2, 719-731 (1998) Zbl 0929.34076 MR 1663336

[11] Lieb, E. H., Thirring, W.: Inequalities for the moments of the eigenvalues of the Schrödinger Hamiltonian and their relation to Sobolev inequalities. In: Studies in Mathematical Physics, Princeton Univ. Press, Princeton, NJ, 269-303 (1976). Zbl 0342.35044 MR 0404846

[12] Simon, B.: The bound state of weakly coupled Schrödinger operators in one and two dimensions. Ann. Phys. 97, 279-288 (1976) Zbl 0325.35029

[13] Weidl, T.: On the Lieb-Thirring constants $L_{\gamma, 1}$ for $\gamma \geq 1 / 2$. Comm. Math. Phys. 178, 135146 (1996) Zbl 0858.34075 MR 1387945

[14] Weidmann, J.: Spectral Theory of Ordinary Differential Operators. Lecture Notes in Math. 1258, Springer, Berlin (1987) Zbl 0647.47052 MR 0923320 